\title{
The Role of Colour Doppler of Carotid Arteries in Cerebrovascular Insufficiency
}

\author{
S Mahbub Pasha ${ }^{1}$ \\ ${ }^{1}$ Assistant Professor, Department of Radiology, SS Institute Of Medical sciences and Research Centre, Davangere, Karnataka.
}

\section{Abstract}

Background: Cerebrovascular accidents constitute a major cause of adult mortality. The principal indication for cerebrovascular Doppler examination is stroke prevention. Colour Doppler sonography is a sensitive method for detection of atherosclerotic plaque and provide considerable information about the extent and severity of plaque as well as the resulting diminution of arterial lumen. The main strengths of Sonography of carotid arteries are patient comfort, lack of risk and accuracy in detecting carotid stenosis. Subjects and Methods: The study was carried out on 40 individuals, among them 20 were symptomatic cases suspected of cerebrovascular insufficiency considered as cases and the other 20 asymptomatic cases with unrelated diseases but having one or the other risk factors for cerebrovascular disease were taken as controls. A detailed clinical history, CNS examination findings and evidence of hypertension, diabetes mellitus, hyperlipidemia and ischemic heart disease were noted. Results: Significant stenosis i.e., $>40 \%$ was found in $12(60 \%)$ of the cases \& in $4(20 \%)$ asymptomatic controls. Peak systolic velocity ratio showed significant stenosis in $12(60 \%)$ of symptomatic cases and in $4(20 \%)$ of asymptomatic controls. Plaque characteristics showed 9 hyperechogenic, 8 calcific, 4 low echogenic and 5 moderate or heterogeneous plaques. Conclusion: The role of carotid Doppler in detecting the site and morphology of atherosclerotic plaque with quantifying the amount of stenosis is very well justified. In addition carotid Doppler can also be used to assess the prognosis in potential symptomatic and asymptomatic patients with one or the other risk factors for cerebrovascular disease.

Keywords: Carotid Doppler, Carotid artery, Peak Systolic Velocity Ratio.

Corresponding Author: Dr. S Mahbub Pasha, Assistant Professor, Department of Radiology, SS Institute Of Medical sciences and Research Centre, Davangere, Karnataka

Received: June 2019

Accepted: June 2019

\section{Introduction}

Stroke or cerebrovascular disease is a major cause of death, ranking third behind only malignancies and cardiovascular disease. Atherosclerosis of the cranial, vessels, leading to cerebral infarction accounts for $80 \%$ of strokes. Intracranial hemorrhage and subarachnoid hemorrhage account for the remainder. It has been conclusively proven that the risk for major stroke is higher in the first three months after T.I.A. ${ }^{[1]}$ It has been seen that $20 \%$ or more of strokes have been heralded by a T.I.A. ${ }^{[2-4]}$ The highest risk of large artery stroke appears to be among patients with, highest degree of carotid stenosis, a history of diabetes, present asymptomatic lesions or a combination of these factors.

Colour Doppler of carotid arteries forms an important part of evaluation of extracranial insufficiency. Accurate diagnosis of haemodynamically significant stenosis is critical to identify patients who would benefit from surgical intervention. The value of a safe, noninvasive and low cost screening test is therefore great. ${ }^{[2]}$

Duplex sonography, combining high-resolution imaging and Doppler Spectrum analysis has provided to be popular, noninvasive, accurate and cost effective means of detecting and assessing carotid disease. Carotid sonography has largely replaced angiography for suspected extiacranial carotid atherosclerosis. ${ }^{[3]}$

If timely atherectomies of the carotid are performed, many strokes cases may be prevented or salvaged. This necessitates evaluation of extracranial. Carotid artery system. Carotid angiography is the gold standard for detecting the severity of carotid disease, but it has its own disadvantages like, it is an invasive and expensive procedure. It carries a risk from contrast medium to the patients and certain amount of morbidity, IVIRI angiography is currently developing rapidly and may ultimately give similar or better results, especially for flow quantification, though at a much higher cost. ${ }^{[4,5]}$

Besides estimating the degree of stenosis, the biggest advantage of sonography is its ability to characterize plaque and identify plaques with higher risk of embolization with high resolution ultrasound, plaque can be characterized into relative risk groups for containing intraplaque hemorrhage which is thought by many to be precursor plaque ulceration.

\section{Subjects and Methods}

The study was carried out on 40 patients. Among them 20 were symptomatic cases suspected of cerebrovascular insufficiency were considered as cases, other 20 
asymptomatic individuals with unrelated diseases having one or more risk factors for cerebrovascular disease on the basis of clinical examination and history were taken as controls.

Method of Collection of Data: The proforma was designed based on the objective of the study and it was pretested and used after modification.

Detailed clinical history and central nervous system examination, findings were noted. Evidence of hypertension, diabetes mellitus, hyperlipidemia and ischemic heart disease were collected. The patients selected as per inclusion criteria were subjected to carotid Doppler examination.

The data gathered from the carotid Doppler examination were as follows:

Peak systolic velocity (PSV) of common carotid artery (CCA) Peak systolic velocity (PSV) of internal carotid artery (ICA). Peak systolic velocity ratio between internal carotid artery and common carotid artery. Measurement of vessel lumen was done from frozen real-time image, the plaque characteristics were seen on real-time image. e Presence of spectral broadening or turbulence were noted down.

Instrument: Sonographic examination of carotid arteries was done by using "HDI 1500" ATL ultrasound system, Bothell WA, USA manufactured by Medisan Co. Ltd., using a high frequency $7 \mathrm{MHz}$ linear array transducer and colour Doppler imaging with 3.5 $\mathrm{MHz}$. The examination was performed with a Doppler angle of $60^{\circ}$ and sample volume of 1 to $5 \mathrm{~mm}$.

Examination Technique: Patient Position: Carotid arteries are examined with patient in the supine position and with the examiner seated at the patient's head. Exposure of the neck is maximized by having the patient drop the ipsilateral shoulder as far as possible. Neck exposure also enhanced by tilting and rotating the head away from the side being examined.

Transducer Position: Generally the posterolateral and far posterolateral positions are most useful for showing the carotid bifurcation and the ICA in longitudinal axis, short axis (transverse) view of the carotid arteries are obtained from an anterior, lateral, or posterolateral approach, depending on which best shows the vessels. The far posterolateral approach often provides the best images of the distal reaches of ICA, and to use this, turn the patient's head far to the contralateral side and place the transducer posterior to the sternomastoid muscle.

\section{Examination Sequence:}

Step-1: Get oriented, choose the transducer position that best displays the carotid vessels in a longitudinal view generally the posterolateral approach is most advantageous. Step-2: Record a velocity spectrum from the CCA. A recording site that is free from disease is preferred, and care should be taken that the sample volume is squarely within the vessel lumen and that the Doppler angle is sufficient to accurately measure the peak systolic velocity.

Step-3: Survey the carotid bifurcation with colour flow imaging. Begin at the clavicle with longitudinal images, proceed to the carotid bifurcation and from there continue into ECA and ICA. Then repeat the process with transverse images. The purpose of this survey is to confirm the patency of the arteries, to identify and localize plaque and associated flow abnormalities and to define the junction of ECA and ICA.

Step-4: Confirm the identity of the ICA and ECA by their Doppler spectral waveforms and by anatomic features. Proper identification of branch vessels is essential, as ECA stenosis usually are not treated surgically, whereas significant ICA stenosis usually treated surgically.

Step-5: With the survey completed and the identity of ICA and ECA confirmed, scrutinize significant areas of plaque formation, documenting with hard copy, the thickness of plaque, the degree of lumen reduction and other plaque features. Transverse images are essential for assessment of plaque thickness, and lumen narrowing. Gray scale images often show plaque features better than 'colour flow images.

Step-6: Most importantly, record angle-corrected velocity spectra in areas at stenosis. Also certain colour flow images that illustrate the location and length of the stenosis, as well as the flow disturbances present in the stenotic and poststenotic regions.

\section{Analysis of Data:}

The collected data was analyzed with the aid of calculator and presented in the form of tables, figures, graphs and diagrams wherever necessary. The findings are discussed in the light of findings of other similar studies conducted elsewhere, based on the objective of the study in the foregoing chapters.

\section{Results}

Table 1: Age wise distribution of cases and controls with significant stenosis

(>40\% stenosis).

\begin{tabular}{|l|l|l|l|l|}
\hline \multirow{2}{*}{ Age ('ears) } & \multicolumn{2}{|l|}{ Cases $(\mathbf{n}=\mathbf{2 0})$} & \multicolumn{2}{l|}{ Controls $(\mathbf{n}=\mathbf{2 0})$} \\
\cline { 2 - 5 } & Number & Percent & Number & Percent \\
\hline$<40$ & 0 & 0.0 & 0 & 0.0 \\
\hline $40-50$ & 1 & 8.3 & 0 & 0.0 \\
\hline $50-60$ & 1 & 8.3 & 1 & 25 \\
\hline $60-70$ & 5 & 41.6 & 1 & 25 \\
\hline $70-80$ & 3 & 25 & 2 & 50 \\
\hline-80 & 2 & 16.6 & 0 & 0.0 \\
\hline
\end{tabular}

Among the cases $(n=20)$, significant stenosis was in 12 numbers. Maximum number of patients with significant stenosis i.e., $>40 \%$ were found in $60-70$ years age group i.e., $5(41.6 \%)$ followed by $70-80$ years age group i.e., 3 (25\%).

In the control group, 4 had significant stenosis were noted. Among them, $2(50 \%)$ were in $70-80$ years age group. 1 $(25 \%)$ was in 50-60 years age group and another $1(25 \%)$ in 60-70 years age group.

Table 2: Plaque characterization in cases and controls Cases (n20) $\quad$ Controls $(\mathbf{n}=\mathbf{2 0})$ 
Pasha; Rale of Calour Dappler of Carotid Arteries in Cerelorovascular Insufficiency

\begin{tabular}{|l|l|l|l|l|}
\hline Characterization & Number & Percent & Number & Percent \\
\hline Low echogenicity & 4 & 15.0 & 5 & 25.0 \\
\hline $\begin{array}{l}\text { Moderate } \\
\text { echogenicity } \\
\text { (heterogeneous }\end{array}$ & 5 & 19.0 & 4 & 20.0 \\
\hline Hyperechogenicity & 9 & 34.0 & 4 & 20.0 \\
\hline Calcified & 8 & 30.0 & 7 & 35.0 \\
\hline
\end{tabular}

Note: 6 patients showed bilateral plaque formation.

In the case group $(n=20)$, majority of the plaque were hyperechogenic in $9(34 \%)$, calcified plaque $8(30 \%)$, moderately echogenic (heterogeneous plaque) $5(19 \%)$ and low echogenic plaque in $4(15 \%)$ of cases.

In the control group $(n=20)$, majority of the plaque were calcified i.e. 7 (35\%) followed by low echogenic plaque in 5 (25\%), moderately echogenic 4 (20\%) and hyperechogenic plaque in $4(20 \%)$.

Table 3: Distribution of cases and controls based on percentage of stenosis

\begin{tabular}{|l|l|l|l|l|}
\hline \multirow{2}{*}{$\begin{array}{l}\text { Percentage of } \\
\text { stenosis }\end{array}$} & \multicolumn{2}{|l|}{ Cases (n20) } & \multicolumn{2}{l|}{ Controls (n=20) } \\
\cline { 2 - 5 } & Number & Percent & Number & Percent \\
\hline$<40 \%$ & 8 & 40.0 & 16 & 80.0 \\
\hline $40-60 \%$ & 5 & 25.0 & 2 & 10.0 \\
\hline $60-80 \%$ & 3 & 15.0 & 2 & 10.0 \\
\hline $80-90 \%$ & 3 & 15.0 & 0 & 0.0 \\
\hline $100 \%$ (total block) & 1 & 5.0 & 0 & 0.0 \\
\hline
\end{tabular}

Among the case group $(n=20), 12$ patients had significant stenosis 60\%. Among these majority were in $40-60 \%$ stenosis group i.e., 5 (25\%) followed by 3 in $60-80 \%$ group $(15 \%)$ and 3 in $80-90 \%$ stenosis group $(15 \%)$ and one patient had total block (complete occlusion) (5\%) and the remaining 8 patients were in $<40 \%$ stenosis group ( $40 \%$ ). In the control group $(\mathrm{n}=20), 16$ individuals were in $<40 \%$ stenosis group (80\%), $2(10 \%)$ had $40-60 \%$ stenosis and the other $2(10 \%)$ had $60-80 \%$ stenosis.

Table 4: Distribution of cases and controls based on peak systolic velocity ratio of ICA/ CCA

\begin{tabular}{|l|l|l|l|l|}
\hline $\begin{array}{l}\text { Peak systolic } \\
\text { velocity ratio of } \\
\text { ICA/ CCA }\end{array}$ & \multicolumn{2}{|l|}{ Cases (n20) } & \multicolumn{2}{l|}{ Controls (n=20) } \\
\cline { 2 - 5 } & Number & Percent & Number & Percent \\
\hline$<1.5$ & 8 & 40.0 & 16 & 80.0 \\
\hline$>1.5$ & 5 & 25.0 & 2 & 10.0 \\
\hline$>1.8$ & 3 & 15.0 & 2 & 10.0 \\
\hline$>3.7$ & 3 & 15.0 & 0 & 0.0 \\
\hline Total block & 1 & 5 & 0 & 0.0 \\
\hline
\end{tabular}

Among the cases $(n=20)$ based on peak systolic velocity ratio of ICA/ CCA, 12 had significant stenosis i.e., 60\%, 5 patients had a ratio of $>1.5(25 \%), 3$ patients had a ratio of $>1.8(15 \%)$ and 3 patients had a ratio $>3.7(15 \%)$ and 1 patient had total block $(5 \%)$.

Among the controls $(n=20)$, only $4(20 \%)$ had significant stenosis, $2(10 \%)$ had a ratio of $>1.5$ and the other $2(10 \%)$ had a ratio of $>1.8$.

\section{Discussion}

In this study, 4 patients $(20 \%)$ had atheromatous plaque in common carotid artery (CCA), 2 on right side (50\%) and 2 on left side $(50 \%)$. 6 patients $(30 \%)$ had atheromatous plaque in the internal carotid artery (ICA), I on right side

$(16 \%), 2$ on left side $(33 \%)$ and 3 on both sides $(50 \%)$. None of the patients had plaque in the external carotid artery (ECA).

Among cases, 8 patients had calcified plaques, 9 patients had hyperechogenicity, 5 moderate echogenicity (heterogenous) and 4 had low echogenicity.

Among the control group, 4 had hyperechogenicity plaque, 4 moderate echogenicity (heterogeneous), 5 low echogenicity and 7 calcified plaques.

The principle arterial pathology detected in the carotid artery disease by. B-mode ultrasonography is "Atherosclerotic plaque". This is seen as "Echogenic" material that encroaches on the arterial lumen and produces a flow void. This was emphasized by Wolverson MK, Bashiti HM, Peterson G et al (1983) and Erickson Si', Middleton WD, Mewiseen MV et a1 (1989) and Erickson Si Mewiseen MW, Foley WD et al (1989). ${ }^{[6]}$

Large plaques may also generate flow disturbances visible on the color Doppler image as stated by Middleton MD, Erickson SJ, Melson GL et a1 (1989). All the above authors have emphasized that plaque echogenicity correlated well with its composition. ${ }^{[7]}$ The bifurcation of the common carotid artery is the commonest source in the extracranial carotid artery, which is in accordance with Philips et al, where they found that the areas of transient reversal of flow, flow separation and eddy formation after referred as "boundary layer separation" corresponds to segments in the carotid bifurcation where atherosclerosis developed first.

Zwiebel WI, Knighton et al (1990) have classified the plaques as follows: 1 . Low echogenicity plaque 2 . Moderate echogenicity (Heterogeneous) 3. Strongly echogenic plaque. 4. Calcified plaques with acoustic shadowing. ${ }^{[8]}$

In the present 'study, 8 patients had calcified plaques, 9 patients with hyperechogenic plaque, and 5 had moderately echogenic plaque. Low Echogenic Plaque: Lusby RI, Ferell LD, Ehrenfed WK et al (1981) have found that "Intraplaque Hemorrhages" occur more commonly in symptomatic patients. They also mentioned that these. "Intraplaque hemorrhages" were associated with fronk ulceration and rapid progression to result in severe luminal narrowing. Fatty plaques, whether homogenous or heterogeneous may result in greater risk of stroke, than the fibrous plaque according to Seeger JM, Klingman N et al (1987), Among the 4 low echogenic plaque lesions noted in our study, "Intraplaque hemorrhage" was not seen in any of the plaques.

Moderately echogenic plaque (heterogeneous plaque): Moderately echogenic plaque (heterogeneous) depends upon the collagen content, which is moderately echogenic and the fat content which is of low echogenicity. The focal hypoechogenicity within this plaques are caused by the presence of thrombus or lipid material within the plaque, according to Zwiebel WI, Knighton et al (1990). In the present study, 5 heterogeneous plaques were noted. ${ }^{[9]}$

Strongly Echogenic (Hyperechoic) Plaque: According to Zwiebel WJ and Knighton et a1, the intraluminal thrombus becomes increasingly echogenic when it becomes retracted and later contracted into fibrous tissue, which suggest the age of the thrombus. They state that a fresh thrombus is virtually anechoic and a very old thrombus is markedly 
echogenic. We came across 9 hyperechogenic plaques, and most of them were suggestive of very old thrombi.

Calcific Plaques: Calcification occurs in plaque in the areas of hemorrhage and necrosis. These calcifications generate strong reflections and distal acoustic shadows. These foci may be focal or diffuse. No correlation exists between the presence of calcification and symptomatology according to Moneto GL, Taylor DC, Nicholls L et al (1987) and Henry ML, Kongable GL, Sevilla EA et al (1988) and Caroll B (1988). But Brown PB, Zwiebel WI, Call CK et a1 (1989) have emphasized that the plaque calcification may have prognostic value and may be useful for selection of patient for Medical or surgical therapy.

Bluth EL, McVay LV, Meritt RB et a (1988) and Reilly M, Lusby RI, Hughes L et al (1985) suggested that the risk of embolization or rapid progression depends upon plaque composition especially if it was heterogeneous, diffuse or focal. We came across 8 calcified lesions. ${ }^{[10]}$

Percentage of significant stenosis with Duplex sonography, among 20 symptomatic cases showed 12 patients $(60 \%)$ had significant stenosis i.e., $>40 \%$ stenosis. Out of 12 patients, 5 had $40-60 \%$ stenosis, 3 had $60-80 \%$ stenosis, the other 3 had $80-90 \%$ stenosis and 1 patient had complete occlusion. The results of 12 out of 20 is consistent with the observations made by Caroll B et al (1989) who found significant involvement of extracranial carotid artery disease between $30-60 \%$.

Among control $(\mathrm{n}=20), 16$ individuals were in $<40 \%$ stenosis group (80\%), only $4(20 \%)$ had significant stenosis, $2(10 \%)$ had $40-60 \%$ stenosis and other $2(10 \%) 60-80 \%$ stenosis.

Peak Systolic Velocity Ratio of ICA/CCA: In the present study among the symptomatic cases $(n=20)$ based on peak systolic velocity ratio of ICA/ CCA, 12 patients had significant stenosis i.e., $60 \%, 5$ patients had a ratio of $>1.5$ $(25 \%), 3$ patients had a ratio of $>1.8(15 \%)$ and 3 patients $>3.7(15 \%)$ and 1 patient had total block.

Among the controls $(n=20), 4(20 \%)$ had significant stenosis, $2(10 \%)$ had $>1.5$ and the other $2(10 \%)$ had $>1.8$.

Bluth EI, Stavros, Marich KW et al defined a velocity ratio greater than 1.8 as an indicator of $60 \%$ or greater and a ratio of 3.7 as an indicator of more than $80 \%$ diameter stenosis.

Garth KE, Caroll BA, Sommer FE (1988) and Oppenheimer DA et al confirmed the validity of 1.5 ratio of peak. Systolic velocity ratio of ICA/CCA as an indicator of $50 \%$ or greater stenosis. They found the ratio more accurate than peak systolic velocity measurements. ${ }^{[11]}$

In this study, peak systolic velocity ratio of ICA/ CCA have been used to define the percentage of significant stenosis as this is more accurate according to a study conducted by Bluth et al.

Robert D Henderson showed that a severely stenosed contralateral ICA can artificially elevate ultrasound, PSV since the effect was greatest when bilateral severe stenosis was present. Caution must be exercised when assessing the degree of ICA stenosis on the basis of ultrasound PSV alone. ${ }^{[12]}$

Based on distribution of cases (n-20) with significant stenosis, 12 patients had significant stenosis. Among them
$41 \%$ were in $60-70$ years age group followed by $70-80$ years age in $25 \%$.

In. the control group 4 individuals with significant stenosis noted, among them $2(50 \%)$ were in $70-80$ years age group and the other $2(25 \%)$ each were in 50-60 years and 60-70 years age groups.

\section{Conclusion}

None of the patients had plaque in external carotid artery (ECA). Based on plaque characteristics, 9 patients had hyperechogenic plaque, 8 calcific plaques, 4 low echogenic plaques and 5 moderate echogenic or heterogeneous plaques in cases while it was 4 hypcrechogenic, 4 moderate echogenic, 5 low echo genic and 7 calcified in the controls. Based on percentage of carotid artery stenosis $12(60 \%)$ of the cases had significant stenosis i.e., $>40 \%$ stenosis. Out of them 5 had $40-60 \%$ stenosis, 3 each had $60-80 \%$ stenosis and $80-90 \%$ stenosis and one patient had complete occlusion. In the control $4(20 \%)$ had significant stenosis, 2 (10\%) had $40-60 \%$ stenosis, 2 (10\%) had $60-80 \%$ stenosis. 0 Based on peak systolic velocity ratio of ICA/ CCA 12 $(60 \%)$ of cases had significant stenosis, 5 cases had a ratio of $>1.5(25 \%), 3$ each had a ratio of $>1.8(15 \%)$ and $>3.7$ $(15 \%)$ and 1 case had total block. While in the controls, 4 $(20 \%)$ had significant stenosis; 2 each $(10 \%)>$ had 1.5 and 1.8. o Based on symptom wise distribution of cases, hemiparesis was found to be the most common presenting symptom in 7 patients $(35 \%)$ and the least presenting symptom was aphasia, dyscalculia in 1 patient $(5 \%)$.

In view of the above findings based on the accuracy of colour Doppler in detecting significant carotid artery stenosis, colour Doppler of carotid artery is recommended in patients with cerebrovascular insufficiency.

\section{References}

1. Longsfeld $\mathrm{M}$ et al. The role of plaque morphology and diameter reduction in the development of new symptoms in asymptomatic carotid arteries. I Vase Surg. 1989; 9: 548-51.

2. Rennie L, Ejrup B, McDowell F, Arterial Bruits in Cerebrovascular disease. Neurology 1964; 14: 751.

3. Feinberg WM, Guidelines for the management of transient ischemic attacks. Circulation, 1994; 89: 2959-2965.

4. Bogousslava I. Clinical predictors of cardiac and arterial lesions in carotid transient ischemic attacks. Lancet, 1994.

5. Asymptomatic carotid atherosclerosis study group: Study design for randomized prospective treatment by carotid endarterectomy for asymptomatic atherosclerosis. STROKE 1989; 20: 844-849.

6. Wilterdink JL. Carotid Duplex ultrasound (CDUS) interpretation in asymptomatic carotid endarterectomy candidates. A patient outcome rather than accuracy based approach. Neurology 1995; 45: A 224.

7. Ricotta JJ et al. A correlative analysis of the human carotid bifurcation by B-mode ultrasound, angiography and pathological examination of endarterectomy specimens. J Vase Surg 1987; 6: 512-520.

8. Pignoli $\mathrm{P}$ et al. Reproducibility of non-invasive ultrasonic measurement of carotid atherosclerosis: The asymptomatic carotid artery plaque study. Stroke 1992; 23: 1062-8.

9. Polak IF et al. Carotid endarterectomy: preoperative evaluation of candidates with combined Doppler sonography and MR angiography. Radiology 1993; 186: 333-8.

10. O'Donnel RF. Correlation of B-mode ultrasound imaging and arteriography with pathologic findings at carotid endarterectomy. Arch Surg 1985; 120: 443-8. 


\section{Pasha; Rale of Calour Dappler of Caratid Arteries in Cereloracascular Insufficiency}

11. Ranval T et al. over-estimation of carotid stenosis: Implications for adults. NEJM, lime 1998; Vol. 338: 1650-1656. carotid endarterectomy. Stroke 1992; 23: 142 .

12. Santhanu R. Srinivasan, Gerald Berenson et al. Association between multiple cardiovascular risk factors and atherosclerosis in young

Copyright: (C) the author(s), publisher. Asian Journal of Medical Radiological Research is an Official Publication of "Society for Health Care \& Research Development”. It is an open-access article distributed under the terms of the Creative Commons Attribution Non-Commercial License, which permits unrestricted non-commercial use, distribution, and reproduction in any medium, provided the original work is properly cited.

How to cite this article: Pasha SM. The Role of Colour Doppler of Carotid Arteries in Cerebrovascular Insufficiency. Asian J. Med. Radiol. Res. 2019;7(1):87-91.

DOI: dx.doi.org/10.21276/ajmrr.2019.7.1.19

Source of Support: Nil, Conflict of Interest: None declared. 